\title{
Lempel-Ziv complexity changes and physiological mental fatigue level during different mental fatigue state with spontaneous EEG
}

\author{
Lian-Yi Zhang ${ }^{1}$, Chong-Xun Zheng ${ }^{2}$ \\ ${ }^{1}$ Electronic Information School of Shanghai Dianji University, Shanghai, 200240, China; ${ }^{2}$ The Key Laboratory of Biomedical Infor- \\ mation Engineering of Ministry of Education, Xi'an Jiaotong University, Xi'an, 710049, China \\ Email: d310zlyi@sohu.com, cxzheng@mail.xjtu.edu.cn
}

Received 6 April 2009; revised 11 May 2009; accepted 15 May 2009.

\begin{abstract}
The objective was to study changes in EEG time-domain Kolmogorov complexity under different mental fatigue state and to evaluate mental fatigue using Lempel-Ziv complexity analysis of spontaneous EEG in healthy human subjects. EEG data for healthy subjects were acquired using a net of 2 electrodes (Fp1 and Fp2) at PM 4:00, AM 12:00 and AM 3:00 in the 24 hours sleep-deprived mental fatigue experiments. It was presented that initial results for eight subjects examined in three different mental fatigue state with 2-channel EEG time-domain Lempel-Ziv complexity computations. It was found that the value of mean Lempel-Ziv complexity corresponding to a special mental state fluctuates within the special range and the value of $C(n)$ increases with mental fatigue increasing for the total frequency spectrum. The result indicates that the value of $C(n)$ is strongly correlative with the mental fatigue state. These results suggest that it may be possible to noninvasively differentiate different mental fatigue level according to the value of $\mathrm{C}(\mathrm{n})$ for particular mental state from scalp spontaneous EEG data. This method may be useful in further research and efforts to evaluate mental fatigue level objectively. It may also provide a basis for the study of effects of mental fatigue on central neural system.
\end{abstract}

Keywords: Lempel-Ziv complexity; mental fatigue; spontaneous EEG; circadiac rhythm

\section{INTRODUCTION}

Fatigue has been a recurrent topic in medicine and psy-

${ }^{*}$ The work is supported by National Natural Science Foundation of China under grant No. 30670534 chology and has recently been attracting much attention. Mental fatigue has been a recurrent topic in medicine and psychology and has recently been attracting much attention. Mental fatigue is a term to cover the deterioration of mental performance due to the proceeding exercise of, mental or physical, activity [1]. Working on cognitively demanding tasks for a considerable time often leads to mental fatigue, which can impact task performance [2]. But the concept of fatigue does not have a clear definition. Thus, prevalence data are always dependent on the particular definition used in the particular paper [3]. Edwards [4] defined fatigue as a "failure to maintain the required or expected force", whereas others [5] have defined it as an inability to "continue working at a given exercise intensity". Gandevia [6] defined fatigue as an exercise-induced loss of power-or force-generating capacity. The physiological definition of fatigue, which is the inability to sustain a specified force output or work rate during exercise, has often been termed "objective fatigue" [7]. D. van der Linden et al [2] defined mental fatigue as a change in psychophysiological state due to sustained performance. This change in psychophysiological state has subjective and objective manifestation, which include an increased resistance against further effort, an increased propensity towards less analytic information processing, and changes in mood. Sustained performance, in this definition, does not necessarily involve the same task but can extend over different tasks that require mental effort, such as fatigue because of a day in the office. Working on cognitive demanding tasks for a considerable time often leads to mental fatigue, which can impact behavior of mental task. In industry, many incidents and accidents have been related to mental fatigue. Therefore, in order to prevent or deal with fatigue related errors it is important to understand the nature of mental fatigue and its specific effects on behavior. The study was conducted to provide some additional insights into mental fatigue and its underlying processes. 
One of the interesting questions in mental fatigue research is in what way cognitive control of behavior changes under fatigue. Lorist [8] used behavioural and EEG-data to study the effects of time-on-task (i.e., mental fatigue) on planning and task switching. The EEGdata of their study showed that with increasing timeon-task there was a reduced involvement of those brain areas that are associated with the exertion of executive control (the frontal lobes). It was reported that subjects protected their performance by spending more effort in the unfavorable conditions: after several hours of work and after continuous work without short rest breaks [1]. The most important change in performance reported by many authors in relation to fatigue is the deterioration of the organization of behavior $[9,10]$. But the ways of evaluating mental fatigue now are based on a subjective sensation rather than on an objective assessment of changes in psychophysiological state, and it does not allow for a clear discrimination between different levels of mental fatigue.

The human brain is one of the most complex systems encountered in nature. Electroencephalogram (EEG) reflects the electrical activity of the central neural system (CNS). Even when the EEGs are analyzed from healthy individuals, they manifest chaos in the nervous systems $[2,8,9,10]$. Although the EEG has limitations with respect to its use as a method for three dimensional anatomical localization of neurofunctional system, it has clear advantages relative to other neuroimaging techniques as a method for continuous monitoring of brain function. It is known that the non-linear dynamic method can explore the specific properties of a system. So there may be some advantages to analyze EEG signals with the nonlinear dynamic method.

Kolmogorov complexity is one of the nonlinear dynamic methods. Intuitively, the complexity of a symbolic sequence reflects an ability to represent a sequence in a compact form based on some structural features of this sequence. To evaluate textual complexity, modifications of the complexity measure by Lempel and Ziv $[11,12,13]$ have been developed. The general approach to estimating the complexity of symbolic sequences (texts) was suggested by A. N. Kolmogorov [14]. He proved that there exists an optimal algorithm or program for the text generation. Lempel-Ziv complexity is the length of the shortest code generating a given sequence. Lempel-Ziv complexity is not a recursive function (i.e. it is not incorporated in a computational scheme). However, for a sequence of finite length, various constructive realizations of non-optimal coding have been developed [14], including applications for DNA analysis $[11,12,13]$. In this paper, the relationship between Lempel-Ziv complexity and different mental fatigue states was investigated.

The aim of this paper was to establish an EEG-based, noninvasive mental fatigue test and to investigate whether mental fatigue could be assessed using Lempel-Ziv complexity measure. Here we present initial results for eight subjects examined in three different mental fatigue state with 2-channel EEG time-domain Lempel-Ziv complexity computations. It was found that the value of mean Lempel-Ziv complexity corresponding to a special mental state fluctuates within the special range and the value of $C(n)$ increases with mental fatigue increasing for the total frequency spectrum.

The rest of the paper is organized as follows. Section 2 explains the methods and algoriyhms proposed in this paper. Mental fatigue experiments are given in Section 3. Section 4 is the results. Discussions and conclusiona are given in Section 5.

\section{METHODS AND ALGORITHM}

Lempel and Ziv proposed measuring the complexity of a sequence by the number of steps in the generating process [14]. The permitted operations here are generation of a new symbol (this operation is necessary at least to synthesize the alphabet symbols) and direct copying of a fragment from the already generated part of the text. Copying implies the search for a prototype (i.e. repeat in a common sense) in the text and extension of the text by attaching the 'prepared' block. c(n) and C(n) both are Lempel-Ziv complexity. The $\mathrm{C}(\mathrm{n})$ is the normalized Lempel-Ziv complexity which does not depend on the length of the sequence when $n$ is large.

The properties of $\mathrm{C}(\mathrm{n})$ for different types of dynamics are: $\mathrm{C}(\mathrm{n})=0$ implies an ordered system, $\mathrm{C}(\mathrm{n})=1$ corresponds to a totally stochastic situation. The higher the $\mathrm{C}(\mathrm{n})$, the closer to a stochastic the system is. So the small value of $\mathrm{C}(\mathrm{n})$ corresponds to the light mental fatigue.

\section{MENTAL FATIGUE EXPERIMENTS}

EEG data were acquired during the task using a net of 26 electrodes. The electrodes were placed at Fp1, Fp2 reference to the 10-20 system to record the EEG data in the experiments. Recordings were made with reference electrode that was pasted to the skin just above the tuber of the clavicle at the right bottom of the Adam's apple by using a high-pass filter of $0.1 \mathrm{~Hz}$ and a low-pass fillter of $100 \mathrm{~Hz}$. Figure 1 shows the placement of electrodes. The impedances of all electrodes were kept below $5 \mathrm{~K} \Omega$. The EEG was acquired using a PL-EEG Wavepoint system and were sampled at about $6 \mathrm{~ms}$ ' interval. The signals were recorded for 5 minutes during the task.

Eight subjects were chosen for the 24 hours sleep- deprived mental fatigue experiments. They were both male and right-handed college students. The work time periods of the college are am 9:00 12:00 and pm 1:00 5:00. All subjects had their normal studing life before the 24 hour experient and the subject was isolated in the experiment. All subjects did not sleep in the afternoon usually. 


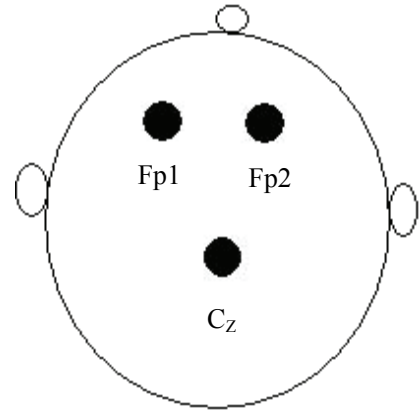

Figure 1. Electrode placement.

The experimental environment was quiet and the temperature of it was around $20 \mathrm{C}^{\circ}$. The subjects were seated in a comfortable chair throughout the experiment. The subjects were asked to simply relax and try to think of nothing in particular.

The light was on during the experiment. The duration of each state was about 5 minutes and the subject's eyes were closed.

State 1: The duration of the experiment time: PM 4:00-4:05.

State 2: The duration of the experiment time: AM 12:00-12:05.

State 3: The duration of the experiment time: AM 3:00-3:05.

Alterations in a person's state of mind are associated with physiological alterations at the neuronal level and sometimes also at the regional brain level [2]. Functionally, central neural system circuitry is complex, consisting of a number of afferent connections, efferent connections and loop ('feedback') connections [8]. Two distinct loops, a motor loop and an association or complex loop, connect basal ganglia with neocortex [9]. The second loop (the association or complex loop) connected caudate with inputs from the cortical association areas and the final output from basal ganglia is projected to the prefrontal cortex. Recent findings have provided considerable evidence that cortex, basal ganglia and thalamus are all linked by the re-entrant circuits [10]. Prefrontal cortex was chosen to place the electrodes. FP1 and FP2 two channels were chosen to record the EEG data. Electrode placement was shown as Figure 1.

\section{RESULTS}

In order to obtain the data of spontaneous EEG signals, a FIR filter with bandpass $0.5-30 \mathrm{~Hz}$ was used. For different sleep-deprived states, the data of two channels (Fp1 and Fp2) corresponding period during experiment was analyzed randomly. The time length of data to be analyzed was 15 seconds. Kolmogorov complexities of 14 continuous but not overlapped period were calculated respectively. The average Kolmogorov complexity under three different fatigue states was shown in Table 1. The results of multiple comparisons were shown in Table 2.
Table 1. Average Kolmogorov complexity under three different fatigue states.

\begin{tabular}{lccc}
\hline \multirow{2}{*}{ Fatigue state } & State 1 & State 2 & State 3 \\
\cline { 2 - 4 } & PM 4:00-4:05 & AM 12:00-12:05 & AM 3:00-3:05 \\
\hline average C(n) & 0.1895 & 0.2569 & 0.2168 \\
\hline
\end{tabular}

Table 2. q test of multiple comparisons.

\begin{tabular}{ccc}
\hline Compare group & $\mathrm{q}(\mathrm{q}$ test) & $\mathrm{P}$ (probability) \\
\hline State 1 and State 2 & $\mathrm{q}(26,2)=8.44$ & $\mathrm{P}<0.01$ \\
State 1 and State 3 & $\mathrm{q}(26,3)=14.18$ & $\mathrm{P}<0.01$ \\
State 2 and State 3 & $\mathrm{q}(26,2)=5.74$ & $\mathrm{P}<0.01$ \\
\hline
\end{tabular}

The results of statistical analysis show that the three state of mental fatigue have significant differences (F2, $26=50.8927, \mathrm{P}<0.01$ ) in general and each state is significantly different from the others. The multiple comparisons showed that sleep may have important influence on Lempel-Ziv complexity or physiologic mental fatigue.

The average of Fp1 and Fp2 channels corresponding to different mental fatigue states changed over time and it was shown in Figure 2. From Figure 2, it can also be seen that the value of mean Lempel-Ziv complexity corresponding to a special sleep-deprived state fluctuates within the special range.

\section{DISCUSSIONS AND CONCLUSIONS}

Human brain is not a stochastic system. $\mathrm{C}(\mathrm{n})=1$ corresponds to a totally stochastic situation. The higher the $\mathrm{C}(\mathrm{n})$, the closer to a stochastic the system is. So the small value of $\mathrm{C}(\mathrm{n})$ corresponds to the light mental fatigue. The work time periods of the college being AM 9:00 12:00 and PM 1:00 5:00, so the cardiac rhythm of the college students corresponds with the work time in day. For most college students, their circadiac rhythms are almost the same.

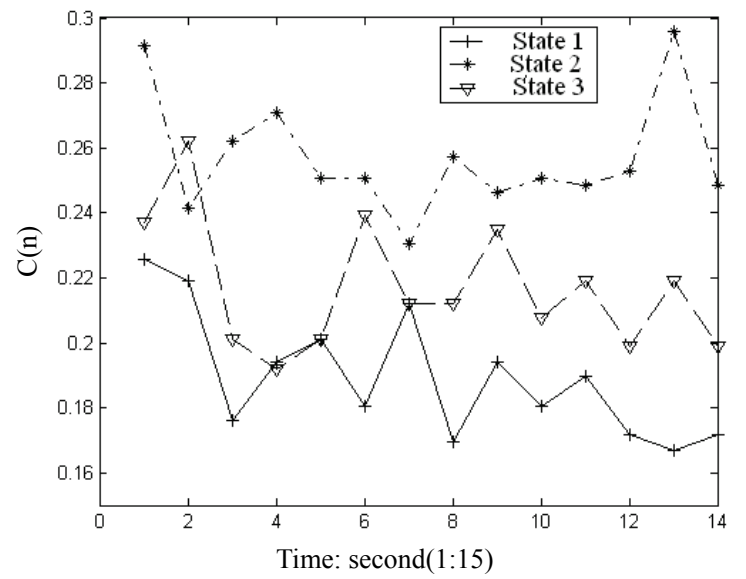

Figure 2. Change of $\mathrm{KC}$ over time under different mental fatigue states. 
In Figure 2, the value of $\mathrm{C}(\mathrm{n})$ under state 2 (AM. 12:00 PM. 12:05) was the largest and the one under state 1 was the smallest. It indicates that, compared with AM. 12:00 or Am. 3:00, college students are more vigorous at PM. 4:00. This is consistent with the fact. At PM 4:00, being in work time of college, study efficiency is higher than in night. It is a normal state that the value of $C(n)$ under state 1 (PM. 4:00 PM. 4:05) was the smallest during all 3 different states. Generally, college students become sober in night and it is wellknown that one is deadly sleepy at midnight and at dawn. Therefore, the value of $\mathrm{C}(\mathrm{n})$ under state 2 (AM. 12:00 AM. 12:05) was larger than the one under state 3 (AM. 3:00 3:05).

In this experiment, the statistical analysis in Section 4 show that the three state of mental fatigue have significant differences $(\mathrm{F} 2,26=50.8927, \mathrm{P}<0.01)$ in general and each state is significantly different from the others. Therefore, the following conclusions can be educed:

1) The value of $C(n)$ is strongly correlative with the mental fatigue state. The value of mean Lempel-Ziv complexity corresponding to a special mental state fluctuates within the special range.

2) The value of $\mathrm{C}(\mathrm{n})$ increases with mental fatigue increasing.

3) It may be possible to differentiate different mental fatigue level according to the value of $\mathrm{KC}$. This method may be useful in further research and efforts to evaluate mental fatigue level objectively. It may also provide a basis for the study of effects of mental fatigue on central neural system.

Fatigue is likely to be an integrated phenomenon with complex interaction among central and peripheral factors, physiological and psychological factors, and so on. All factors appear to mutually influence each other. The method proposed in this paper includes only two EEG channels of one cortical region and one parameter. For those that mental states are very close according to time or mental fatigue level, it may be difficult to differentiate them by the method proposed in this paper.

Our results suggest that the value of mean Lempel-Ziv complexity corresponding to a special mental state fluctuates within the special range and the value of $\mathrm{KC}$ increases with mental fatigue increasing. The results re- ported here do not replace the results obtained with the traditional test and other techniques, but supplement them. In summary, these results suggest that it may be possible to noninvasively detect, and even eventually distinguish different level of mental fatigue from scalp EEG data. This could be very helpful for safety in production. These findings are preliminary and need to be further studied in a large population base.

\section{REFERENCES}

[1] T. F. Meijman, (1997) Mental fatigue and the efficiency of information processing in relation to work times, International Journal of Industrial Ergonomics, 20 (1), 31-38.

[2] D. Linder, M. Frese, and T. F. Meijman, (2003) Mental fatigue and the control of cognitive process: Effects on perseveration and planning, Acta Psychologica, 113 (1), 45-65.

[3] T. Akerstedt, A. Knustsson, P. Westterholm, et al., (2004) Mental fatigue, work and sleep, Journal of Psychosomatic Research, 57, 427-433.

[4] R. Edwards, (1981) Human muscle function and fatigue, Ciba. Found. Symp., 82, 1-18.

[5] F. Booth and D. Thomason, (1991) Molecular and cellular adaptation of muscle in response to exercise: perspectives of various models, Physiol. Rev., 71, 541-585.

[6] S. C. Gandevia, (2002) Spinal and supraspinal factors in human muscle fatigue, Physiol. Rev., 81, 1725-1789.

[7] R. B. Layzer, (1998) Asthenia and chronic fatigue syndrome, Muscle Nerve, 21(12), 1609-1611.

[8] M. M. Lorist, (2000) Mental fatigue and task control: Planning and preparation, Psychophysiology, 37, 1-12.

[9] D. E. Broadbent, (1979) Is a fatigue test possible now? The society lecture, Ergonomics, 12, 1277-1290.

[10] D. H. Holding, (1983) Fatigue, In G. R. J. Hockey (ed), Stress and fatigue in human performance, New York: Wiley.

[11] V. D. Gusev, V. A. Kulichkov, and O. M. Y. Chupakhina, (1991) Complexity analysis of genomes. I. Complexity and classification methods of detected structural regularities, Mol. Biol. (Mosk)., 25(6), 825-834.

[12] V. D. Gusev, L. A. Nemytikova, and N. A. Chuzhanova, (1999) On the complexity measures of genetic sequences, Bioinformatics, 15(7), 994-999.

[13] X. Chen, S. Kwong, and M. A. Li, (1999) Compression algorithm for DNA sequences and its applications in genome comparison, Genome Inform. Ser. Workshop Genome Inform., 10(1), 51-61.

[14] A. Lempel and J. Ziv, (1976) On the complexity of finite sequences, IEEE Trans. Inf. Theory, 22(1), 75-81. 